\title{
Biometric Authentication with Face Recognition using Principal Component Analysis and Feature based Technique
}

\author{
Onifade, F.W. Olufade \\ Computer Science Department \\ University of Ibadan, Ibadan
}

\author{
Adebayo, J. Kolawole \\ Computer Science Department \\ Oduduwa University, lle-Ife
}

\begin{abstract}
In today's fast paced networked world, the need to maintain the security of information or physical property is becoming both increasingly important and increasingly difficult. Recently, a ground breaking technology; biometrics, which is still a subject of growing research became available to allow verification of "true" individual identity. This is the focal point of our work where a face recognition system is implemented. We implemented an authentication system based on face recognition. We trained the images using principal component analysis and then combine with a feature based technique. For the feature based technique, we extract some key features including the red, green and blue colours of the eyes, the width and height of the eyes etc and ratios between them. We computed weights for each image based on these features and record the weights in the database for each subject in the database. We finally combine these feature weights with the weights computed from the principal component analysis and used it as the final weight to perform recognition. The system achieved a good recognition result.
\end{abstract}

\section{General Terms}

Face Recognition

\section{Keywords}

Principal component analysis, Feature based technique, Biometric, authentication, Threshold.

\section{INTRODUCTION}

In today's fast paced networked world, the need to maintain the security of information or physical property is becoming both increasingly important and increasingly difficult. From time to time various technology-based crimes e.g. credit card fraud, computer break-in by hackers, or security breaches in a company or government building are being perpetrated. In most of these crimes, the criminals take advantage of a fundamental flaws in the conventional access control systems, this is achievable because the systems do not grant access into such facilities by "who people are", that is what makes them distinct but by "what people have", that is what they possesses such as ID cards, keys, passwords which does not really define individuals as being unique personalities, therefore, if anyone steals, duplicates, or acquires these identity means, he or she will be able to access other peoples data or personal property.

Recently, a ground breaking technology which is still a subject of growing research became available to allow verification of "true" individual identity. This technology is called "biometrics" and it is the focal point of our research work wherein a face recognition system is implemented. Authentication refers to the verification that you are who you say you are i.e. genuinely proving ones identity [1]. These are commonly applied when for example users tries to log onto a network, perform an on-line transaction etc, the authentication process verifies to ascertain the users identity. Usually, this is done by providing the system with a characteristic or combination of characteristics that are associated with ones identity. Obviously, biometrics authenticates humans more reliably when compared to other methods of authentication.

Biometric has been defined an automated method of identity verification or identification based on the principle of measurable physiological or behavioral characteristics such as finger-print, iris pattern, facial characteristics or a voice sample [2]. As such, in any authentication applications, a user is either accepted as being the true identity or rejected as being an impostor, that is, the output is a binary response, yes or no.

Face recognition has gained much attention in recent years and has become one of the most successful applications of image analysis. It is one of the few biometric methods that possess the merits of both high accuracy and low intrusiveness. It has the accuracy of a physiological approach without being intrusive; it is hands-free and continuous while being accepted by most users because of its low-intrusiveness. A typical application is to identify or verify the person of a given face in still or video images.

The important applications of face recognition are in areas of biometrics i.e. computer security and human computer interaction [3].

Several approaches to modelling facial images are Principal Component Analysis, Local Feature Analysis, Linear discriminant analysis and Fisher face which are all based on dimensionality reduction. Also neural networks, elastic bunch graph theory, 3D morphable models and multi-resolution analysis are some other techniques usually used. This work focuses on the biometric authentication using face recognition. Our preferred technique is the principal component analysis; a holistic approach, among the various techniques available. Principal component analysis is based on Karhunen-Loeve transform and is our choice because of its simplicity, learning capability, robustness to small changes in the face image, speed and lesser computational overhead when compared to some other techniques.

Our work is different from the existing works because of the application of several pre-processing algorithms to serve as multiple filters for the image in order to reduce the false acceptance rate (FAR) and false rejection rate (FRR) in our proposed system. We also enhanced our system with a feature based technique. Also, our system works in an interactive time thus giving the user the real-time experience. The 
remaining part of this work is divided into four parts. The following section is the literature review, followed by the methodology and then the section showing our results. The last section contains our conclusion and future works.

\section{LITERATURE REVIEW}

Reference [4] has a comprehensive survey of different face recognition techniques which include detailed description and classification of the algorithms both for still and video based recognition and should be consulted for further review.

Most works in computer recognition of faces has focused on detecting individual features such as the eyes, nose, mouth, and head outline, and defining a face model by the position, size, and relationships among these features. Such approaches have proven difficult to extend to multiple views and have often been quite fragile, requiring a good initial guess to guide them. Research in human strategies of face recognition, moreover, has shown that individual features and their immediate relationships comprise an insufficient representation to account for the performance of adult human face identification. Nonetheless, this approach to face recognition remains the most popular one in the computer vision literature

One of the first works in face recognition was in [5], where a face recognition technique which focuses on detecting important facial features or key-points such as the eye corners, nose tips, mouth corners and chin edge was implemented. Relative distances between facial key-points were measured and a feature vector constructed to describe each face. These feature vectors were then used in comparing known faces in the database to unknown probe faces.

In reference [6], semi-automated face recognition with a hybrid human-computer system that classified faces on the basis of fiducially marks entered on photographs by hand was implemented. Parameters for the classification were normalized distances and ratios among points such as eye corners, mouth corners, nose tip, and chin point. Also reference [7], attempted to measure similar features automatically. In it, a linear embedding algorithm that used local feature template matching and a global measure of fit to find and measure facial features was described. In reference [8] the work done by Fischler et al was improved on, their work focused on deformable templates. Deformable templates are parameterized models of the face and its features in which the parameter values are determined by interactions with the face image. In [9] and [10], an associative network with a simple learning algorithm that can recognize face images and recall a face image from an incomplete or noisy version input to the network was described and was later extended in [11] by using nonlinear units and training the system by back propagation. In [12], all steps of the recognition process were automated, using a top-down control strategy directed by a generic model of expected feature characteristics.

The holistic approach makes use of template matching and identifies faces using global representations i.e. the whole face is seen as one object [13], it then extract features from the whole face region. In this approach, as in the previous approach, the pattern classifiers are applied to classify the image after extracting the features.

A method of extracting features in a holistic system is by applying statistical methods such as Principal Component Analysis (PCA) to the whole image. PCA can also be applied to a face image locally; in that case the approach is not holistic. Irrespective of the methods being used, the main idea is the dimensionality reduction. A method usually used is the Eigenface Method by Turk and Pentland [14] which is based on the Karhunen-Loeve expansion. Their work is motivated by the ground breaking work of Sirovich and Kirby [15],[16] and is based on the application of Principal Component Analysis to the human faces.

When we talk of principal component analysis, the focus is dimensionality reduction, this is achieved by extracting the desired number of principal components of the multidimensional data in which case the first principal component is the linear combination of the original dimensions that has the maximum variance and the $\mathrm{n}^{\text {th }}$ principal component is the linear combination with the highest variance, subject to being orthogonal to the n-1 first principal components. We are considering a way of extracting the relevant information of a face while also capturing the variation in a collection of face images (usually the face database to be trained) and thus encode those variations efficiently in order for us to be able to compare it with other similarly encoded faces.

Reference [17] proposed a method using PCA which detects the head of an individual in a complex background and then recognizes the person by comparing the characteristics of the face to those of known individuals. Also, in [18], PCA was used for coding and compression for video streams of talking heads. They suggest that a typical video sequence of a talking head can often be coded in less than 16 dimensions. A similarity measure for direct image matching based on a Bayesian analysis of image deformations was proposed in reference [19]. In their work, they modeled two classes of variation in object appearance: intra-object and extra-object. The probability density functions for each class were then estimated from training data and finally used to compute a similarity measure based on the posteriori probabilities. They further present a novel representation for characterizing image differences using a deformable technique for obtaining pixelwise correspondences. Their representation, based on a deformable 3D mesh in XYI-space, was experimentally compared with two simpler representation i.e. intensity differences and optical flow. They claimed a good result.

In reference [20] the use of PCA and Gabor Filters was suggested. Firstly, Gabor Filters, Log Gabor filters and Discrete wavelet transform were used to extract facial features from the original image on predefined fiducial points. PCA was then used to classify the facial features optimally and reduce the dimension. The approximation coefficients in discrete wavelet transform was extracted and was then used to compute the face recognition accuracy instead of using all the coefficients. They suggest the use of combining these methods in order to overcome the shortcomings of PCA. Also, [21] argued that, when raw images are used as a matrix of PCA, the eigenspace cannot reflect the correlation of facial feature well, as original face images have deformation due to in-plane, in-depth rotation, illumination and contrast variation. Also they argue that, they have overcome these problems using Gabor Filters in extracting facial features.

Reference [22] implemented a feature based system; they used a fairly simple fingerprint which includes eye and skin colour, ratios of distances between prominent facial features such as eyes, mouth, nose and chin, and absolute and relative values of width and height of the face and the eyes. The system described the overall geometrical configuration of face features by a vector of numerical data representing position and size of main facial features. First, they extracted eyes coordinates. The interocular distance and eyes position was used to determine size and position of the areas of search for 
face features. WEKA was used to rank and determine the facial features that contribute most to recognition. They claimed that their experimental results showed that their method is robust, valid for numerous kinds of facial image in real scene, works in real time with low hardware requirements and the whole process is conducted automatically as applicable for an amber alert system they implemented.

A feature-based technique for face recognition in which eigenface was applied to sub-images (eye, nose, and mouth) was implemented in [23]. In it, they applied a rotation correction to the faces in order to obtain better results.

\section{METHODOLOGY}

\subsection{Proposed System Overview}

The proposed system though primarily based on the PCA technique, is enhanced by being combined with a feature based technique. Our aim is to get the advantage of the two techniques and thus a more efficient system. Our system passes through different stages after acquisition and before recognition, the first being the extractions of some facial features which we think are very important. Here, we select some features and use them as distinct fingerprints for each individual image in the database, we then compute some weight for each fingerprint and total the aggregate weight for each subject in the database, and the score is then labelled for each subject.

After extraction of the needed features, we apply the PCA to the same image set in the database, this gives us some weight descriptors for each image after the eigenface has been computed and thus gives us the possibility of adding the total weight score we got for each subject from their fingerprints to the new score computed based on their weight from the eigenface. For any probe image we are recognizing, we also make it to go through the above steps, such that the important features are also extracted and scored and the eigenface computed. We finally add the two weight scores i.e. from the eigenface computation and the features extracted and then compare the score with the aggregate scores in the database, if it matches the score of any subject in the database, we recognize it as known. The steps are detailed as below:

1) Face database formation phase: Acquisition and preprocessing / normalization of face images are done here, then the images are stored in the database. Training is performed on the images in this database and their corresponding eigenfaces and eigenvalues created. Our system is designed to operate on $128 \times 128$ images in the database, to perform image size conversions and enhancements on face images; we have pre-processing steps for normalization which rescale all images to $128 \times 128$. Here, we also perform histogram equalization and background removal to improve face recognition performance. For each face acquired, we have two entries in the database such that one is for the image itself while the other is the weight vectors computed after training is done; this weight vector is used to compute the ultimate weight for each image.

2) Training phase: Training of the images in the database is done based on the PCA technique; principal component analysis is performed on the image set in order to calculate the eigenfaces which are then stored for later use, keeping only the $\mathrm{M}$ images that correspond to the highest eigenvalues. These $\mathrm{M}$ eigenfaces define the $\mathrm{M}$-dimensional "face space". As new faces are experienced, the eigenfaces can be updated or recalculated. The corresponding distribution in the $\mathrm{M}$ dimensional weight space is calculated for each face database member, by projecting its face image onto the "face space" spanned by the eigenfaces. The corresponding weight vector of each image in the database is then updated and recognition can be performed after we might have added the weights computed from the feature based. The algorithm below depicts the steps taken when computing the eigenface and weights using the PCA.

1. Let's assume the face images in our database is $x_{1}, x_{2}, x_{3}$, $\mathrm{x}_{4} \ldots \ldots, \mathrm{x}_{\mathrm{M}}$ then we find the mean image which is

$$
\Psi=\frac{1}{m} \sum_{n=1}^{m} X_{n}
$$

2. Next, we have to know how each face differs from the mean image above like this $\Phi_{i}=X_{i}-\Psi$

This set of very large vectors is then subject to principal component analysis, which seeks a set of $\mathrm{M}$ orthogonal vectors, $U_{n}$, which best describes the distribution of the data. The $\mathrm{k}^{\text {th }}$ vector, $\mathrm{U}_{\mathrm{K}}$, is chosen such that the eigenvalues

$$
\begin{gathered}
\lambda_{k}=\sum_{n=1}^{m}\left(\boldsymbol{U}_{K}^{T} \Phi_{n}\right)^{2} \quad \text { which is also subject to } \\
\text { eigenvector } \boldsymbol{U}_{I}^{T} \boldsymbol{U}_{\boldsymbol{k}}=\delta_{i \boldsymbol{k}}=\left\{\begin{array}{cc}
\boldsymbol{1}_{2} & \text { if } 1=k \\
0, & \text { otherwise }
\end{array}\right\} \text { where the }
\end{gathered}
$$
vectors $\mathbf{U}_{\mathrm{K}}$ and scalars $\lambda_{\mathrm{K}}$ are the eigenvectors and eigenvalues, respectively of the covariance matrix $\mathrm{C}$ of the training images depicted as

\section{$\mathrm{C}=\frac{1}{m} \sum_{n=1}^{m} \Phi_{n} \Phi_{n}^{T}=A A^{T}$}

In essence we are calculating the covariance matrix $\mathrm{C}$.

3. The matrix $\mathrm{A}=\left[\phi_{1}, \phi_{2}, \phi_{3} \ldots \ldots . \phi_{\mathrm{m}}\right]$. The covariance matrix $\mathrm{C}$, however is $\mathrm{N}^{2} \times \mathrm{N}^{2}$ real symmetric matrix, and determining the $\mathrm{N}^{2}$ eigenvectors and eigenvalues is an intractable task for typical image sizes. We need a computationally feasible method to find these eigenvectors.

Following these analysis, we can then construct the $\mathrm{M} \times \mathrm{M}$ matrix $\mathrm{L}=\mathrm{A}^{\mathrm{T}} \mathrm{A}$ where $\mathrm{L}_{\mathrm{mn}}=\phi_{\mathrm{m}}^{\mathrm{t}} \phi_{\mathrm{n}}$ and then find the $\mathrm{M}$ eigenvectors, $\mathrm{V}_{\mathrm{i}}$ of $\mathrm{L}$. These vectors determine linear combinations of the $\mathrm{M}$ training set of face images to form the eigenfaces $U_{i}$. Which we represent as $U_{I}=\mathbf{U}_{\mathbf{l}}=$ $\sum_{k=1}^{m} V_{I K} \Phi_{K}$ where $\mathrm{I}=1 \ldots \ldots$. The associated eigenvalues allow us to rank the eigenvectors based on how useful they are in characterizing the variation among the images. It should be noted that the eigenvalues is a value associated with the eigenfaces/eigenvector $U_{I}$ These eigenvalues are used to construct weights which are kept in the database with the label of that image i.e. the name of the person. Recognition is delayed till after extracting the important features and computing weight for the image with our feature based technique.

\subsection{Feature Extraction and Ranking}

Humans have always identify faces perfectly despite the marked similarity of faces as spatial patterns, this is possible because of our ability to extract invariant structural information from the transient situation of faces such as changing hairstyles, emotional expression, and facial motion effect. In fact, features are the basic elements for object recognition. Therefore, identifying and extracting effectively used features in human face recognition may be very useful. In this work, in addition to the eigenface method applied above, we carefully chose some features that we found to be very important and that mostly differs from person to person e.g. we found that the distances between the eyes, nose, and mouth were not useful as they vary little between people and 
thus we do not consider them for usage, likewise we found the eye and skin colour, ratios of distances between prominent facial features, and absolute and relative values of width and height of the face and the eyes to be useful.

For each facial image, we create fingerprint of some features, these fingerprints were determined based on our analysis of facial images and the variations between them. The list of our finally chosen features includes:

- red, green, and blue values of the eye colour

- ratios between the red and the green values of the eye colour denoted as RG

- ratios between the green and the blue values of the eye colour denoted as GB

- ratios between the red and the blue values of the eye colour denoted as RB

- the width and height of the eye

- the ratio between the width and height of the eye

- the ratio between the distance between the two eyes and the distance between the eye-line and the nosetip

- the width and height of the face

- the ratio between the width and height of the face

- the RGB values of the skin colour

- The number of lines passing around the chin. Here, we used Hough transform to determine this.

After determining the features to be used, we extract them based on the methods proposed in [22], compute the values for them, and record those values in the database. The procedure is stated as below:

1) We first determine the location of the two eye pupils and extract the eye colour by computing the average red, green, and blue values of each pixel in a determined area encompassing the eye pupil, excluding pixels which represent a skin colour.

2) We also calculate the ratios between these values, that is, the red and green, green and blue, and red and blue values of the eyes and record them in the database for each image. It must be noted that for each record stored, we label it according to the name of the person whose face is in the database and we are currently processing.

3) Using the location of the eye pupils, we check a small rectangular region surrounding the eye pupil for the outer most left, right, bottom, and top pixels which do not represent a skin colour. This gives us the width and height of each eye, however, each eye may differ slightly in width and height and thus we will sum up the widths and heights of the two eyes and divide by two to obtain the average width and height of the eye, this is also recorded in the database as well as the ratio between the original widths and heights. The diagram below shows the procedure

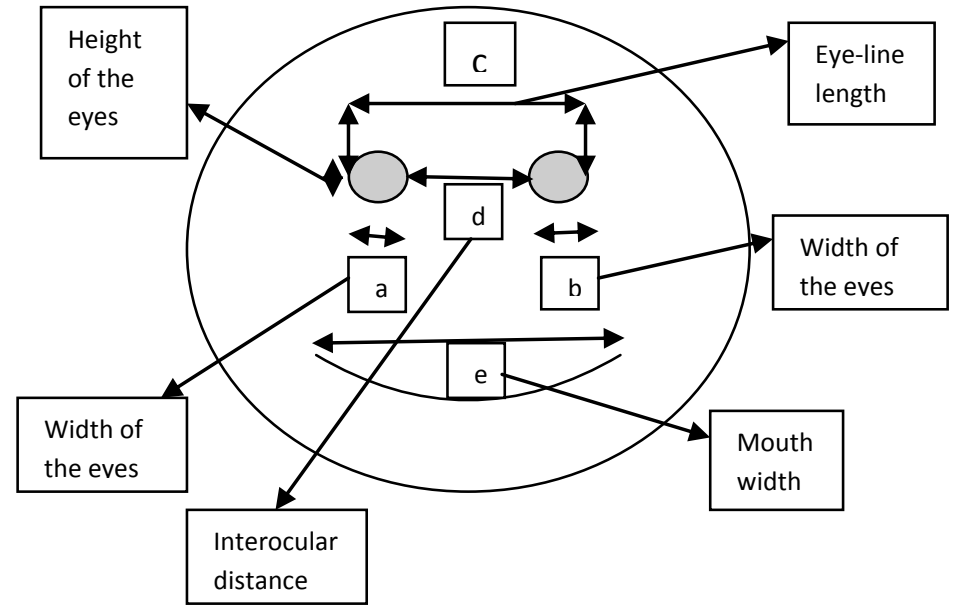

Fig 1: Eye and head midpoint localization and Left and right eye measurement.

Average eye height $(\mathrm{H})$

Average eye width (W)

Ratio between the width and height of the eye $=\mathrm{H} / \mathrm{W}$

4) After that is done, we make a "face mask" by locating the nose and a rectangular shape in which, for every pixel, we have to check whether it represents a skin pixel. We also used a smaller range allowed for a pixel to be recognized as a skin pixel in order to avoid picking up hair colour as skin.

5) We check whether each pixel is at a specific distance from the nose (this is shorter near the eyes, longer near the chin and the forehead). If this is true, and this pixel is also a skin pixel, we include it in the face mask. Since we are only interested in ending the most extreme points of the bounding box for the face, we keep track of the rightmost, leftmost, bottom and top pixels of the face mask only.

6) We also compute the width and height of the face, as well as the ratio between them, and record it in the database for each of the subject to be trained.

7) We then compute the red, green, and blue values for each skin pixel and calculate their average, which we also record in the database.

8) Hough Transform is performed to detect

a) All regions in the image which resemble straight lines, then

b) The angle of each line is determined.

Since each image has been labeled according to the name of each person in the database and the above procedure has been performed on all the images, this gives us the chance of recording all the records taken with the name of each person in the database which also correspond to the label of the image. We simply choose some values as multiplier for all the features extracted, these are just assumed values given for the features in order to construct the weights. These values must be the same for every image we process and are as below: 
a) We multiply each of the red, green, and blue values of the eye colour by a multiplier of 10 .

b) The ratios between the red and the green values of the eye colour denoted as RG by 20

c) The ratios between the green and the blue values of the eye colour denoted as GB by 30

d) The ratios between the red and the blue values of the eye colour denoted as RB with 40

e) The width and height of the eye with 50 each

f) The ratio between the width and height of the eye by 60

g) The ratio between the distance between the two eyes and the distance between the eye-line and the nosetip by 70

h) The width and height of the face by 80 each

i) The ratio between the width and height of the face by 90

j) The RGB values of the skin colour by 100

k) The number of lines passing around the chin by 5 .

The total feature extracted weight thus becomes:

Total_feature_weight $=\mathrm{a}+\mathrm{b}+\mathrm{c}+\mathrm{d}+\mathrm{e}+\mathrm{f}+\mathrm{g}+\mathrm{h}+\mathrm{i}+\mathrm{j}+\mathrm{k}$ as computed from above.

We then total the sum of these values to get the aggregate weight score (Total_feature_weight) which we then record in the database also with the name of the person in the image. Finally, we look at the weight computed by the eigenface technique above for each of the image labelled identically and add the value to the corresponding weight aggregate from the features extracted, this we then keep in the database as our final weight ranking of each image.

The proposed system can be represented diagrammatically as shown in figure 2, detailing all the major interactions between the sub-systems.

\subsection{Recognition and learning phase}

The focus of any authentication system is to distinguish between a true identity and a fake identity. Usually, the true identity is authenticated while the fake is denied. Thus, the goal of our proposed system is to recognize and authenticate any probe issued by the user, the probe image is acquired from the camera, rescale to the default size and normalized for any inconsistency such as lighting effect etc.

We then apply PCA to construct its weight vector with eigenface, thus,

a) The new face image $\left(X_{\text {new }}\right)$ is transformed into its eigenface components (i.e. projected onto "face space") by a simple operation $\omega^{\mathrm{K}}=\mathrm{U}^{\mathrm{T}}{ }_{\mathrm{k}} \cdot\left(\mathrm{X}_{\text {new }}-\Psi\right)$. This is simply subtracting the mean image from the probe image. For $\mathrm{k}=1 \ldots \mathrm{M}^{\prime}$. This describes a set of point by point image multiplications and summations, operations performed at approximately frame rate on current image processing hardware.

b) The weights form a feature vector, $\Omega^{\mathrm{T}}{ }_{\text {new }}=\left[\omega_{1} \omega_{2} \ldots \ldots \omega\right.$ M] that describes the contribution of each eigenface in representing the probe face image, treating the eigenfaces as a basis set for face images and its size is $\left(\mathrm{M}^{\prime} \mathrm{x} 1\right)$.

c) The eigenvalues corresponding to the feature vector is then computed,

d) After this, we also make the probe image to go through our feature extractor in order for us to compute our facial features weight as done above when computing for each image in the database.

e) We then add the weight score from the PCA to the score just computed from the extracted features in order to get the ultimate weight for that image as computed for all the face database members.

f) This is our classifier stage, in which we compare the ultimate weight computed for the probe image to the weights already computed for all the image database members. i.e. let the ultimate weights computed and stored with the labels of each person in the database be $\mathrm{T} 1, \mathrm{~T} 2 \ldots \ldots . \mathrm{Tn}$ and the ultimate weight for the probe image be $\mathrm{T}_{\text {probe }}$ then this procedure is depicted as below

$$
\begin{aligned}
& \text { For } j=1 \text { to } n \\
& \text { If } T_{j}==T_{\text {probe }} \\
& \text { 1) Accept image asrecognized } \\
& \text { 2) Display } T_{j} \text { as a recognized face database member } \\
& \text { 3) Authenticate } T_{j} \\
& \text { Else } j=j+1 \\
& \text { End If }
\end{aligned}
$$
End for.

Thus if there is an image in the face database member that is similar to the acquired image within that threshold i.e. if a hit occurs (such that there is a match whereby the new weight coincide with any of the weight already computed in the database) we check for that particular subject, displayed the image of that subject and output such probe as being recognized i.e. the face image is classified as "known" and thus authenticated. Otherwise, a miss has occurred and the face image is classified as "unknown". After being classified as unknown, this new face image is added to the face database with its corresponding weight vector for later use (we take this to be learning to recognize).

The next section contains the results obtained from the experiments conducted.

\section{RESULTS}

We present the results obtained for the recognition rate on the proposed face recognition system. The system was trained with a database of 15 subjects each having 5 images under different light intensity, scale and head orientations. The testing was carried out with 45 images as test/probe images of the subjects.

The training of the database member was carried out successfully and the test cases constructed differently to measure the accuracy level of our system under the following conditions:

1) Histogram equalized database members for illumination independence

2) Un-equalized database member to ascertain the performance under different lighting conditions. 
3) Different threshold values i.e. we increased the threshold by $10 \%$ for each test iteration, starting from original value of 3.00 as the threshold.

4) Recognition performance when the number of images in the trained database per each person is varied i.e. is used per subject.

5) All the images are automatically rescaled to 128 by 128 in order to get a constant scaling.

The algorithm was successfully implemented using JAVA, the training and simulation was performed on 32 bit operating system, an AMD Turion ${ }^{\mathrm{TM}} 64$ X2 Mobile Technology with a processor speed of $1.98 \mathrm{GHz}$ and memory of $2 \mathrm{~GB}$ to provide valuable results. The tables below show the results obtained.

Table.1. Table showing the recognition rate when all images are equalized. we performed the experiment when 1, 3 and 5 images

Table.3. Table showing the recognition rate when the threshold value is being varied. It should be noted that the images are also equalized.

\begin{tabular}{llllll}
\hline $\begin{array}{l}\text { Total } \\
\text { Number } \\
\text { of } \\
\text { Images }\end{array}$ & $\begin{array}{l}\text { Total no } \\
\text { of } \\
\text { subjects }\end{array}$ & $\begin{array}{l}\text { Number } \\
\text { of } \\
\text { training } \\
\text { images } \\
\text { per } \\
\text { subject }\end{array}$ & $\begin{array}{l}\text { Threshold } \\
\text { Value used }\end{array}$ & $\begin{array}{l}\text { Number } \\
\text { of test } \\
\text { images } \\
\text { per } \\
\text { subject }\end{array}$ & $\begin{array}{l}\text { Average } \\
\text { recognition } \\
\text { rate }\end{array}$ \\
\hline $\mathbf{7 5}$ & 15 & 5 & 3.00 & 3 & $79.8 \%$ \\
\hline $\mathbf{7 5}$ & 15 & 5 & 3.50 & 3 & $81.1 \%$ \\
\hline $\mathbf{7 5}$ & 15 & 5 & 4.00 & 3 & $83.2 \%$ \\
\hline $\mathbf{7 5}$ & 15 & 3 & 3.00 & 3 & $70.2 \%$ \\
\hline $\mathbf{7 5}$ & 15 & 3 & 3.50 & 3 & $72.5 \%$ \\
\hline $\mathbf{7 5}$ & 15 & 3 & 4.00 & 3 & $73.2 \%$ \\
\hline $\mathbf{7 5}$ & 15 & 1 & 3.00 & 3 & $66.3 \%$ \\
\hline $\mathbf{7 5}$ & 15 & 1 & 3.50 & 3 & $67.2 \%$ \\
\hline $\mathbf{7 5}$ & 15 & 1 & 4.00 & 3 & $67.8 \%$ \\
\hline
\end{tabular}

\begin{tabular}{lllll}
\hline $\begin{array}{l}\text { Total } \\
\text { Number } \\
\text { of }\end{array}$ & $\begin{array}{l}\text { Total no } \\
\text { of } \\
\text { Images }\end{array}$ & $\begin{array}{l}\text { Number } \\
\text { of } \\
\text { training } \\
\text { images } \\
\text { per } \\
\text { subject }\end{array}$ & $\begin{array}{l}\text { Number } \\
\text { of test } \\
\text { images } \\
\text { per } \\
\text { subject }\end{array}$ & $\begin{array}{l}\text { Average recognition } \\
\text { rate (success) }\end{array}$ \\
\hline $\mathbf{7 5}$ & 15 & 5 & 3 & $86.7 \%$ \\
\hline $\mathbf{7 5}$ & 15 & 3 & 3 & $81.9 \%$ \\
\hline $\mathbf{7 5}$ & 15 & 1 & 3 & $66.3 \%$ \\
\hline
\end{tabular}

Table.2. Table showing the recognition rate when images are not equalized

\begin{tabular}{lllll}
\hline $\begin{array}{l}\text { Total } \\
\text { Number } \\
\text { of }\end{array}$ & $\begin{array}{l}\text { Total } \\
\text { no of } \\
\text { subjects }\end{array}$ & $\begin{array}{l}\text { Number } \\
\text { of } \\
\text { training } \\
\text { images } \\
\text { per }\end{array}$ & $\begin{array}{l}\text { Number } \\
\text { of test } \\
\text { images } \\
\text { per } \\
\text { subject }\end{array}$ & $\begin{array}{l}\text { Average } \\
\text { recognition } \\
\text { rate (success) }\end{array}$ \\
& & 5 & 3 & $79.8 \%$ \\
\hline $\mathbf{7 5}$ & 15 & 3 & 3 & $70.2 \%$ \\
\hline $\mathbf{7 5}$ & 15 & 3 & 3 & $52 \%$ \\
\hline
\end{tabular}

\section{CONCLUSION}

In this work, a face recognition based authentication system has been implemented. We combined two major techniques in order to increase the proposed system's efficiency i.e. principal component analysis was used to compute some weight for each image which was then added to the weights computed by our feature based approach.

We also applied some pre-processing steps like the gray scaling, histogram equalization, background removal and automatic rescaling of all images concerned in order to achieve illumination and scale invariance. Our system works well for the authentication system we have implemented and our training of images and recognition done relatively fast. In fact, as against some of the existing systems, the proposed system gives a good recognition result even with a one image is trained per subject. The results above further shows the average recognition rate for each of the experiments conducted. Future works can extend this work to include pose invariance and robustness to facial details such as beard and glasses worn by subject which are not covered in our system.

\section{ACKNOWLEDGMENTS}

We acknowledge the constructive criticism and constant advice of senior faculty members of computer science department of university of Ibadan. 


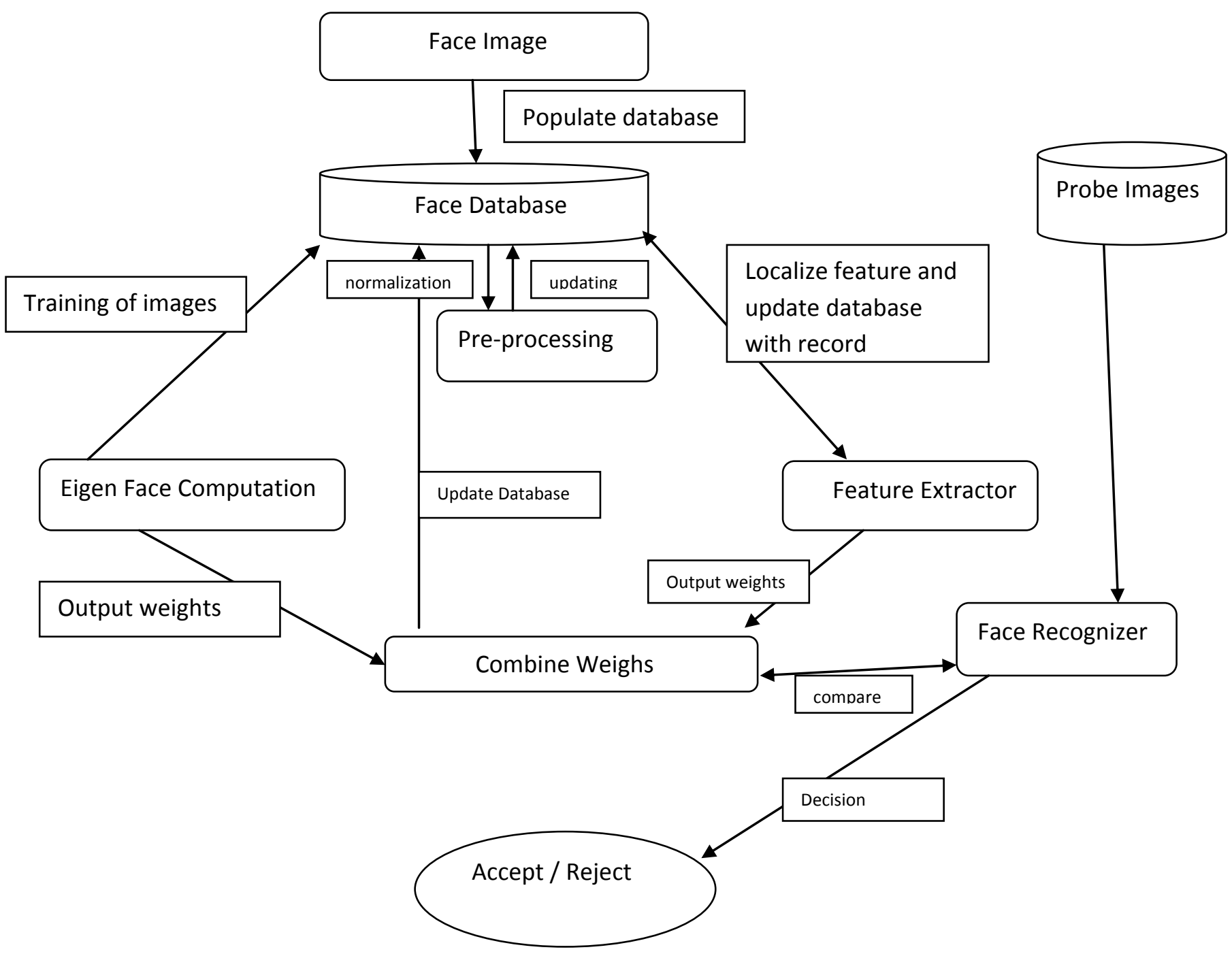

Fig 2: The Proposed Face Recognition Model.

\section{REFERENCES}

[1] V. Matyas and Z. Riha, Biometric authenticationsecurity and usabilityy, Faculty of informatics, masaryic university Brno, Czech Republic. 2008.

[2] V. Matyas and Z. Riha, "Towards reliable user authentication through biometrics". IEEE security and privacy journal, 2007.

[3] D. Kresmir, G. Mislav, and L. Panos, "Appearance Based statistical method for face recognition" in $47^{\text {th }}$ international sysmposium, ELMAR 2005, Zedar, Croatia . June 2005.

[4] W. Zhao, R. Chellapa, and P. J Phillips, "Face Recognition: A literature survey ," in Technical Report, University of ,Maryland, 2000.

[5] F.Galton, "Personal identification and description 1,1 Nature, pp.173-177,21 June1888

[6] W.W Bledsoe, "The model method in facial recognition", Panoramic Research Inc. Palo Alto, CA, Rep. PRI:15, (August 1966).
[7] M.A. Fischler, and R.A Elschlager, "The representation and matching of pictorial structures", IEEE Trans. on Computers, c-22.1, (1973).

[8] A.L Yuille, D. S Cohen, and P. W. Hallinan, , "Feature extraction from faces using deformable templates", Proc. of CVPR, (1989).

[9] T. Kohonen, "Self-organization and associative memory", Berlin: Springer- Verlag, (1989).

[10] T. Kohonen, and P. Lehtio, "Storage and processing of information in distributed associative memory systems", (1981).

[11] M. Fleming and G. Cottrell, "Categorization of faces using unsupervised feature extraction", Proc. of IJCNN, Vol. 90(2), (1990).

[12] T. Kanade, "Picture processing system by computer complex and recognition of human faces", Dept. of Information Science, Kyoto University, (1973).

[13] J. Huang, "Detection strategies for face recognition using learning and evolution" $\mathrm{PhD}$ thesis, George Mason University. ,May 1998. 
[14] M. Turk, and A. Pentland., "Eigenfaces for recognition", Journal of Cognitive Neuroscience, Vol. 3, pp. 71-86, (1991).

[15] M. Kirby., and L. Sirovich., "Application of the Karhunen-Loeve procedure for the characterization of human faces", IEEE PAMI, Vol. 12, pp. 103-108, (1990)

[16] M. Kirby., and L. Sirovich., "Low-dimensional procedure for the characterization of human faces", J. Opt. Soc. Am. A, 4, 3, pp. 519-524, (1987).

[17] S.J. Lee, S.B. Yung, J.W. Kwon and S.H. Hong, "Face detection and recognition using PCA". Pg84-87. IEEE. TENCOM, 1999.

[18] J.L. Crowley and K. Schwerdt, "Robust tracking and compression for video communication". Pg 2-9. IEEE transaction on pattern recognition. 1999.

[19] B. Moghaddam, C. Naster and A. Pentland, "A Bayesian similarity measure for deformable image matching", Image and Vision computing, vol 19, may 2001.

[20] D. Murugan, S. Murugam,K. Rajalakshmi and T.I Manish, "Performance evaluation of face recognition using Gabor filter, Log Gabor filter and Discrete Wavelet Transform. International Journal of computer science and information technology. Vol 2, no 1, feb 2010.

[21] B. Moghaddam. Principal manifolds and bayesian subspaces for visual recognition. IEEE Transactions on Pattern Analysis and Machine Intelligence, 24(6):780\{788, June 2002.

[22] S. Cagnoni, A.Poggi, "A modular eigenspace approach to face recognition" Pg 490-495. IEEE Transaction on pattern recognition. 1999.

[23] S. Cagnoni, A.Poggi, "A modified modular eigenspace approach to face recognition" Pg 490-495. IEEE Transaction on pattern recognition. 1999. 